\title{
First application of an axial speed of sound measurement technique in the monitoring of tendon healing
}

\author{
C. VERGARI* ${ }^{*}$, P. POURCELOT $\dagger$, B. RAVARY-PLUMIOËN $\dagger$, A.G. DUPAYS $\dagger$, \\ S. JACQUET $\dagger$, J.M. DENOIX $\dagger$, D. MITTON $\ddagger$, P. LAUGIER $\S$ and N. CREVIER-DENOIX $\dagger$ \\ $\dagger$ USC INRA ENVA BPLC, Ecole Nationale Vétérinaire d'Alfort, Maisons-Alfort, France \\ ¥Université de Lyon, F-69622, Lyon, France; Ifsttar, LBMC, UMR_T9406, F-69675, Bron ; \\ Université Lyon 1, Villeurbanne. \\ § UPMC Université Paris 6, UMR CNRS7623, LIP, Paris, F-75005 France.
}

Keywords: quantitative ultrasound; speed of sound; ultrasonography; tendon

\section{Introduction}

Ultrasonography is the reference technique to assess acute tendon lesions. However, as lesions start healing, they present a relatively normal mean echogenicity although tendon is not yet healed and its elasticity has not recovered.

Tendon axial speed of sound (SOS), currently applied to non-invasively evaluate tendon loads [1], is a quantity directly depending on tendon elastic properties, so it represents a good candidate to evaluate tendon health and quality.

Therefore the objective of this study was to evaluate if tendon SOS can quantify the evolution of tendon lesions as they heal and to investigate its relation with quantitative characteristics of tendon ultrasonographic (US) images.

\section{Methods}

12 French Trotters (2-4 years old, $429 \pm 39 \mathrm{Kg}$ average body mass) participated in a clinical trial testing a regenerating agent on tendon lesions. A region of interest (ROI) was defined in the metacarpal area of the right limb superficial digital flexor tendon (SDFT) of each horse. A core lesion was surgically induced (as approved by an ethical committee) in the ROI and, for symmetry, in the same area of the opposite limb.

The SDFT axial SOS was measured in the ROI with the probe and fixation protocol described in [1]. Transversal US images (using an Aloka Alpha10 Prosound, 7.5 MHz linear probe) of each ROI were acquired by trained clinicians.

SOS measurements (defined as the velocity of the first arriving signal with signals at $1 \mathrm{MHz}$ ) were performed during sessions of 6 series of straight line walks (30 strides total for each horse per session) on an asphalt pavement. Those sessions took place before the surgical induction (SI) of the core lesion and about each month, for 3 months, starting 15 days after SI. SOS values of each horse were calculated by averaging the maximal SOS values (corresponding to the maximal SDFT load at the walk) measured in each session (finding a coefficient of variation inferior to $0.8 \%$ ).

US images were acquired immediately after each SOS measurement session. The operator adjusted the ultrasound machine's parameters in order to optimize the SDFT brightness and contrast, and traced the tendon's contour. Two superficial layers 2 and $4 \mathrm{~mm}$ deep (equal to and twice the order of magnitude of the wavelength for a speed of sound of about $2000 \mathrm{~m} / \mathrm{s}$ at $1 \mathrm{MHz}$ ), $10 \mathrm{~mm}$ wide, were extracted, in each image, in the zone where the SOS measuring probe had been placed (Figure 1). The mean and standard deviation (SD) of the gray scale distribution in these 2 layers were then calculated. Echogenicity's SD was chosen as a preliminary approach to evaluate tendon's architecture. It was hypothesized that this SD would be higher in normal tendon tissue because of the ultrasonic reflection between intact tendon fibers fascicles, compared to an injured tendon when the fascicular organization is altered.

\section{Results and Discussion}

Average SOS for each measurement session is reported in Table 1. SOS decreased in all tendons after the SI, and it continued decreasing in 11 out of 12 tendons 1.5 months after the SI. At 2.5 months, half of the horses showed an increase in SOS, while at 3.5 months 11 out of 12 horses showed an increase (Figure 2), although no one reached its initial SOS value.

Echogenicity's mean and standard deviation of both the 2 and $4 \mathrm{~mm}$ layers had a less characteristic evolution (Table 1): these values generally decreased between the examinations before and 15 days after the SI, but then their evolution did not show a general tendency as the lesion healed (i.e., their values increased or decreased, depending on the horse and from one measurement session to the other).

However, correlations $(\mathrm{p}<0.05)$ were found between SOS measurements and both the echogenicity's mean $(r=0.30)$ and SD $(r=0.52)$ of the $4 \mathrm{~mm}$ layer, when the pooled data of all 5 


\begin{tabular}{|l|l|l|l|l|l|}
\hline Session & Before SI & $\begin{array}{l}15 \text { days } \\
\text { after SI }\end{array}$ & $\begin{array}{l}1.5 \text { months } \\
\text { after SI }\end{array}$ & $\begin{array}{l}2.5 \text { months } \\
\text { after SI }\end{array}$ & $\begin{array}{l}3.5 \text { months } \\
\text { after SI }\end{array}$ \\
\hline Mean SOS \pm SD $[\mathrm{m} / \mathrm{s}]$ & $2179 \pm 31$ & $2107 \pm 49$ & $2049 \pm 64$ & $2045 \pm 66$ & $2066 \pm 67$ \\
\hline Mean echogenicity \pm SD & $117.6 \pm 18.8$ & $84.2 \pm 9.0$ & $111.2 \pm 9.9$ & $89.2 \pm 12.0$ & $95.1 \pm 13.2$ \\
\hline SD of echogenicity \pm SD & $34.7 \pm 2.8$ & $29.9 \pm 2.8$ & $32.0 \pm 2.9$ & $28.6 \pm 2.2$ & $30.0 \pm 1.9$ \\
\hline
\end{tabular}

Table 1 Average speeds of sound (SOS) values, average means and average standard deviations of tendon US images echogenicity, in a $4 \mathrm{~mm}$ superficial layer, before and after the surgical induction (SI) of a core lesion

sessions for all horses were considered. SOS was also found correlated with the echogenicity's SD of the $2 \mathrm{~mm}$ layer $(\mathrm{r}=0.39)$.

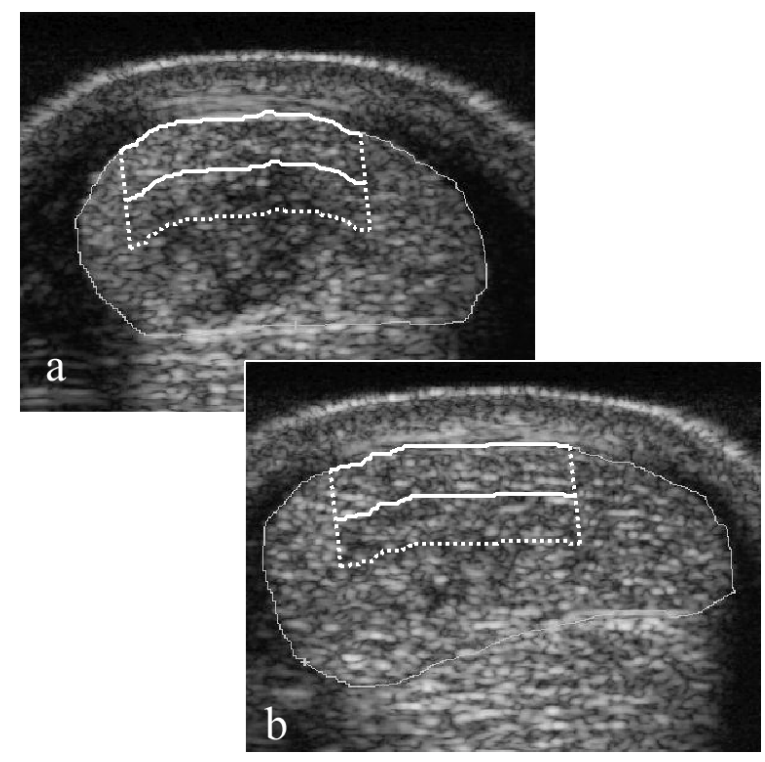

Figure 1 Typical example of the US images of the superficial digital flexor tendon, (a) 15 days and (b) 3.5 months after the surgical induction of a lesion (horse \#12). The solid and dashed lines are the 2 and $4 \mathrm{~mm}$ thick layers, respectively; the layers are centered in the SOS measurement zone.

These data suggest that the evolution of SOS values for each horse bears information which is not available with US images of healing lesions, as SOS continues to decrease even when the mean superficial echogenicity does not show a clear tendency. The SOS decrease suggests that even the superficial part of the tendon, initially intact or only slightly injured, can be affected by the core lesion evolution. This could be due to the progressive infiltration of the initially normal peripheral tendon tissue (i.e. around the core lesion) by an inflammatory healing tissue. The global correlation observed with the echogenicity's SD suggests that SOS is affected by tendon's heterogeneity that could reflect architectural alterations.

The stronger correlation found between SOS and the properties of the $4 \mathrm{~mm}$ layer which, often comprehends a part of the core lesion, was also informative regarding the depth of tendon tissue likely explored by the technique.

\section{Conclusions}

Tendon SOS could be an informative mean to characterize and follow up the healing of a tendon lesion: the results of this preliminary study suggest that the tendon elastic properties are the weakest at about 2 months after the injury onset (assuming that the mass density of the explored area remains the same).

However, further studies are needed in order to robustly model the relation between tendon's mechanical properties and SOS. Moreover, the depth of the tissue actually explored by the ultrasound waves forming the first arriving signal should be properly checked, both at $1 \mathrm{MHz}$ and at lower frequencies, which would extend the area explored by this technique.

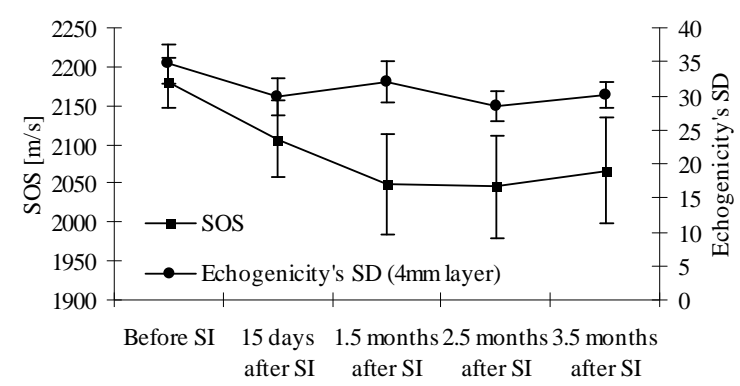

Figure 2 Average speed of sound values $( \pm$ SD) in all horses before and after the surgical induction (SI) of a core lesion, confronted with average echogenicity's SD of the $4 \mathrm{~mm}$ tendon layer.

\section{References}

[1] Crevier-Denoix N., Pourcelot P., Ravary B., Robin D., Falala S., Uzel S., Grison A.C., Valette J.P., Denoix J.M., and Chateau H., Influence of track surface on the equine superficial digital flexor tendon loading in two horses at high speed trot. Equine Veterinary Journal, 41, 257-261.

[2] Laugier P., Haiat G., Bone Quantitative Ultrasound. Springer, 2011.

\section{Acknowledgements}

The authors are grateful to the Direction Générale de l'Enseignement et de la Recherche, the Région Basse Normandie and the Institut National de la Recherche Agronomique for financial support. This study was funded by the Agence Nationale de la Recherche. 